\title{
Photo-stability of $\mathrm{CsPbr}_{3}$ perovskite quantum dots for optoelectronic application
}

\author{
Junsheng Chen ${ }^{1,2}$, Dongzhou Liu ${ }^{3,4}$, Mohammed J. Al-Marri ${ }^{5}$, Lauri Nuuttila ${ }^{6}$, Heli Lehtivuori ${ }^{6}$ \\ and Kaibo Zheng ${ }^{1,5^{*}}$
}

\begin{abstract}
Due to their superior photoluminescence (PL) quantum yield (QY) and tunable optical band gap, all-inorganic $\mathrm{CsPBr}_{3}$ perovskite quantum dots (QDs) have attracted intensive attention for the application in solar cells, light emitting diodes (LED), photodetectors and laser devices. In this scenario, the stability of such materials becomes a critical factor to be revealed. We hereby investigated the long-term stability of as-synthesized $\mathrm{CsPbBr}_{3}$ QDs suspended in toluene at various environmental conditions. We found light illumination would induce drastic photo-degradation of $\mathrm{CsPbBr}_{3} \mathrm{QDs}$. The steady-state spectroscopy, transmission electron microscopy (TEM), and X-ray diffraction (XRD) verified that $\mathrm{Cs} \mathrm{PbBr}_{3}$ QDs tend to aggregate to form larger particles under continuous light soaking. In addition, decreasing PL QY of the QDs during light soaking indicates the formation of trap sites. Our work reveals that the main origin of instability in $\mathrm{CsPbBr}_{3}$ QDs and provides reference to engineer such QDs towards optimal device application.
\end{abstract}

Keywords: perovskite, quantum dots, photo-stability

\section{INTRODUCTION}

Recently, all-inorganic cesium lead halide perovskite colloidal quantum dots $\left(\mathrm{CsPbX}_{3} \mathrm{QDs}, \mathrm{X}=\mathrm{Cl}, \mathrm{Br}, \mathrm{I}\right)$ have attracted broad research interest in the application of solar cells, light emitting diodes (LED), lasers, and photo-detectors because of their broad band absorption, high photoluminescence (PL) quantum yield (QY) $\Phi_{\mathrm{PL}}$ up to $90 \%$, narrow PL emission bandwidth $[1,2]$, spectrally tunable emission across the whole visible range by simply changing the composition and size of the QDs, and favorable optical gain together with low-threshold spontaneous emission [1-8].

On the other hand, the stability of $\mathrm{CsPbX}_{3}$ QDs still remains unclear, which is one critical factor for their device application. In fact, the instability of $\mathrm{APbX}_{3}$ (where $\mathrm{A}=$ $\mathrm{CH}_{3} \mathrm{NH}^{3+}$, or $\mathrm{HC}\left(\mathrm{NH}_{2}\right)^{2+} ; \mathrm{X}=\mathrm{Br}^{-}, \mathrm{I}^{-}$, and/or $\mathrm{Cl}^{-}$) perovskite towards moisture, light and heat has long been considered as the main restriction for the real industrialization of corresponding devices [4,9-12]. Such instability is also troublesome for fundamental studies [4]. Some previous investigation has tested the stability of $\mathrm{CsPbBr}_{3}$ QDs $[4,13]$. However, the environmental conditions (i.e., temperature, light condition, and atmosphere) have not been considered in those studies, which are critical in the working devices (e.g., solar cells, LEDs and photo-detectors). In particular, the stability of colloid perovskite QDs solution is an important parameter for the production and storage of $\mathrm{CsPbBr}{ }_{3}$ QDs inks in printed electronics and optoelectronics devices. This motivated us to carry out a detailed stability study of $\mathrm{CsPBr}_{3}$ QDs in toluene under various conditions by spectroscopic methods. The results revealed the intensive degradation of $\mathrm{CsPbBr}{ }_{3}$ QDs under light excitation above their optical band-edge reflecting as aggregation of the nanocrystals and introducing surface or interface trap sites.

\section{EXPERIMENTAL SECTION}

$\mathrm{Cs} \mathrm{PbBr}_{3}$ colloidal QDs were prepared using the method developed by Kovalenko and co-workers [3]. In brief, $0.814 \mathrm{~g}$

\footnotetext{
${ }^{1}$ Department of Chemical Physics and NanoLund, Lund University, Lund 22100, Sweden

${ }^{2}$ State Key Laboratory of Molecular Reaction Dynamics, Dalian Institute of Chemical Physics, Chinese Academy of Sciences, Dalian 116023, China

${ }^{3}$ College of Science, Agricultural University of Hebei, Baoding 071001, China

${ }^{4}$ College of Physics Science \& Technology, Hebei University, Baoding 071002, China

${ }^{5}$ Gas Processing Center, College of Engineering, Qatar University, Doha, Qatar

${ }^{6}$ University of Jyväskylä, Department of Physics, Nanoscience Center, Jyväskylä, Finland

*Corresponding author (email: kaibo.zheng@chemphys.lu.se)
} 
$\mathrm{Cs}_{2} \mathrm{CO}_{3}$ (Sigma-Aldrich, 99\%) was mixed with $40 \mathrm{~mL}$ 1-octadecene (Sigma-Aldrich, 90\%) and $2.5 \mathrm{~mL}$ oleic acid (OA, Sigma-Aldrich, 90\%), and then heated to $120^{\circ} \mathrm{C}$ for $1 \mathrm{~h}$ under vacuum. Consequently, the mixture was heated up to $150^{\circ} \mathrm{C}$ for $30 \mathrm{~min}$ under $\mathrm{N}_{2}$ atmosphere. The obtained Cs-oleate was kept in the glove box and heated up to $100^{\circ} \mathrm{C}$ before using. $0.689 \mathrm{~g} \mathrm{PbBr}_{2}$ (Sigma-Aldrich, 99.999\%) and $10 \mathrm{~mL} 1$-octadecene were heated up to $120^{\circ} \mathrm{C}$ under vacuum for $1 \mathrm{~h}$. Afterwards $0.5 \mathrm{~mL}$ dry oleylamine (OAm, Sigma-Aldrich, 80-90\%) and $0.5 \mathrm{~mL}$ OA were added into the solution. The temperature of the solution was increased to $180^{\circ} \mathrm{C}$ followed by rapid injection of $0.4 \mathrm{~mL}$ Cs-oleate solution. After injection the reactant was cooled by ice-water bath immediately. The ice-water cooled crude solution was centrifuged at 6,500 rpm for $10 \mathrm{~min}$. After centrifugation, the supernatant was discarded and the particles were redispersed in toluene (Sigma-Aldrich, 99.8\%). In order to obtain QDs with narrow size distribution, the crude solution was centrifuged at $2500 \mathrm{rpm}$ for $5 \mathrm{~min}$ again to exclude the aggregated large clusters. After this centrifugation, the supernatant was collected and diluted to $\sim 26 \mathrm{nmol} \mathrm{L}^{-1}$ with toluene. The diluted samples were then divided into seven portions and stored under different conditions (summarized in Table 1) for stability test.

The UV-vis absorption spectra were measured using a PerkinElmer Lambda 1050 spectrophotometer. The steady-state fluorescence spectra were recorded by a standard spectrofluorometer (Spex1681) with excitation at $430 \mathrm{~nm}$. The PL QYs of samples S1-S7 were calculated relative to standard fluorescein dye (PL QY $=95 \%$, in 0.1 mol L ${ }^{-1} \mathrm{NaOH}$ aqueous solution) $[14,15]$. Time-resolved PL measurements were performed using a time-correlated single-photon counting (TCSPC) device (PicoQuant). A pulsed diode laser, triggered externally at $2.5 \mathrm{MHz}$, was used to excite the sample at $438 \mathrm{~nm}$ (at fluence of 0.5 $\mu \mathrm{J} \mathrm{cm}^{-2}$ ). The pulse duration of the laser was about $200 \mathrm{ps}$

Table 1 Sample storage conditions

\begin{tabular}{cccc}
\hline Sample & Temp. & Atmosphere & Light condition $^{\text {a) }}$ \\
\hline S1 & Room temp. & $\mathrm{N}_{2}$ & Dark \\
S2 & Room temp. & Air & Dark \\
S3 & $4^{\circ} \mathrm{C}$ & Air & Dark \\
S4 & Room temp. & $\mathrm{N}_{2}$ & LED \\
S5 & Room temp. & Air & LED \\
S6 & Room temp. & Air & LED+filter1 \\
S7 & Room temp. & Air & LED+filter2 \\
\hline
\end{tabular}

a) LED lamp is from ORLED, model: RL18 $\left(50 \mathrm{~mW} \mathrm{~cm}^{-2}\right), 440 \mathrm{~lm}$; filter 1 is a $300-500 \mathrm{~nm}$ band pass filter; filter2 is a $550 \mathrm{~nm}$ long pass filter.
[16]. The emitted photons were detected with a $450 \mathrm{~nm}$ long band pass filter and focused onto a fast avalanche photodiode (SPAD, Micro Photon Device) with response time less than 50 ps.

The size of $\mathrm{CsPbBr}_{3}$ QDs was characterized by transmission electron microscopy (TEM, Jeol JEM 1400) at an accelerating voltage of $80 \mathrm{kV}$. XRD patterns were recorded in the range $13^{\circ}<2 \theta<25^{\circ}$ using $0.02^{\circ}$ steps on a 4 -circle Huber diffractometer with a $\mathrm{Si}$ (111) double crystal monochromator with a $12 \mathrm{keV}(0.1 \mathrm{~nm}) \mathrm{X}$-ray light source. XRD measurements were carried out at MCX-Powder diffraction beamline in Elettra synchrotron light source, Italy. The samples were dried under vacuum and transferred to caption tape for the measurement.

\section{RESULTS AND DISCUSSION}

\section{Stability test by steady-state spectroscopies}

In order to investigate the stability of $\mathrm{CsPbBr}{ }_{3}$ QDs at different temperature, atmosphere and light irradiation conditions, we stored the samples at seven different conditions (see Table 1). Absorption spectra (Fig. 1) can be utilized to monitor the evolution of QD concentration in the solution overtime. The absorption spectra of samples S1-S3 stored under dark condition (corresponding to Figs $1 \mathrm{a}, \mathrm{b}$ and $\mathrm{c}$ ) remain unchanged. However, the absorption spectra of sample S4 and S5 with light irradiation (corresponding to Figs $1 \mathrm{~d}$ and e) exhibit pronounced change at different time. This reflects as reduced absorbance together with gradually increasing offset below the absorption edge due to the light scattering (Figs 1d and e). According to Beer-Lambert law, the absorbance is proportional to the concentration of $\mathrm{CsPbBr}_{3}$ QDs at linear region. Therefore, we can conclude the concentration of $\mathrm{Cs} \mathrm{PbBr}_{3}$ QDs in sample S4 and $\mathrm{S} 5$ is continuously decreasing. Moreover, the absorption edges of all the samples initially at $503 \mathrm{~nm}(2.46 \mathrm{eV})$ are gradually red-shifted (Fig. 1f). Among them the spectral shift in sample $S 5$ is most pronounced $(10 \mathrm{~nm})$, which makes the absorption edge close to the bulk material (2.25 $\mathrm{eV}, 550 \mathrm{~nm})[17,18]$. According to the well-accepted theory in semiconductor QDs, such red shift of the absorption edge and reduced absorption coefficient of QDs are the fingerprint of the weakening of the quantum confinement, which indicates the size increasing of QDs towards the exciton Bohr diameter of the bulk materials (12 nm) $[18,19]$. The following morphological characterization can further verify $\mathrm{CsPBr}_{3}$ QDs tend to aggregate to larger size particles under light irradiation.

Besides the optical absorption we also monitored the PL 

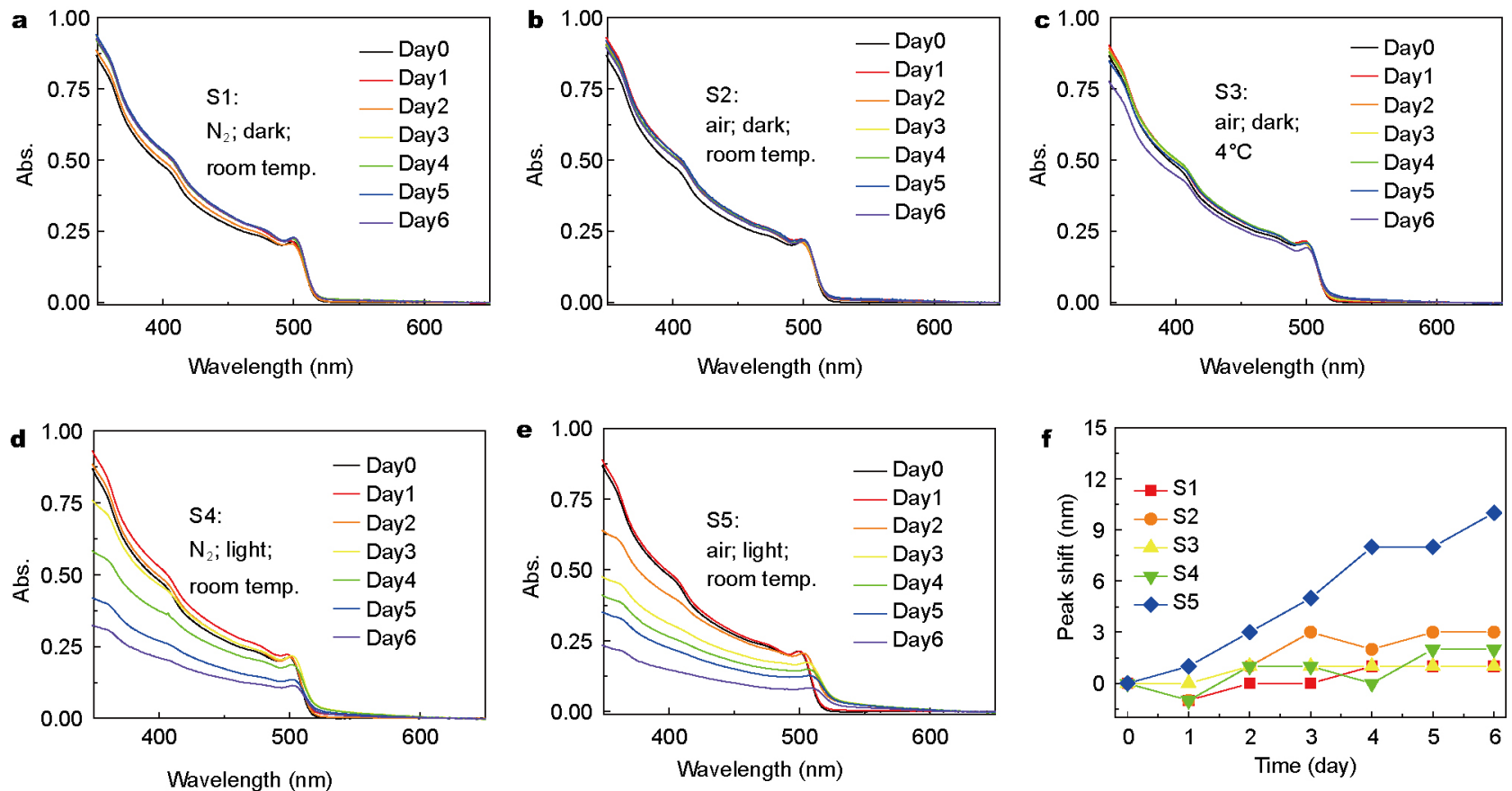

Figure 1 Absorption spectra of samples S1-S5 (a-e) at different time, and (f) absorption edge shift for samples S1-S5 during the stability test.
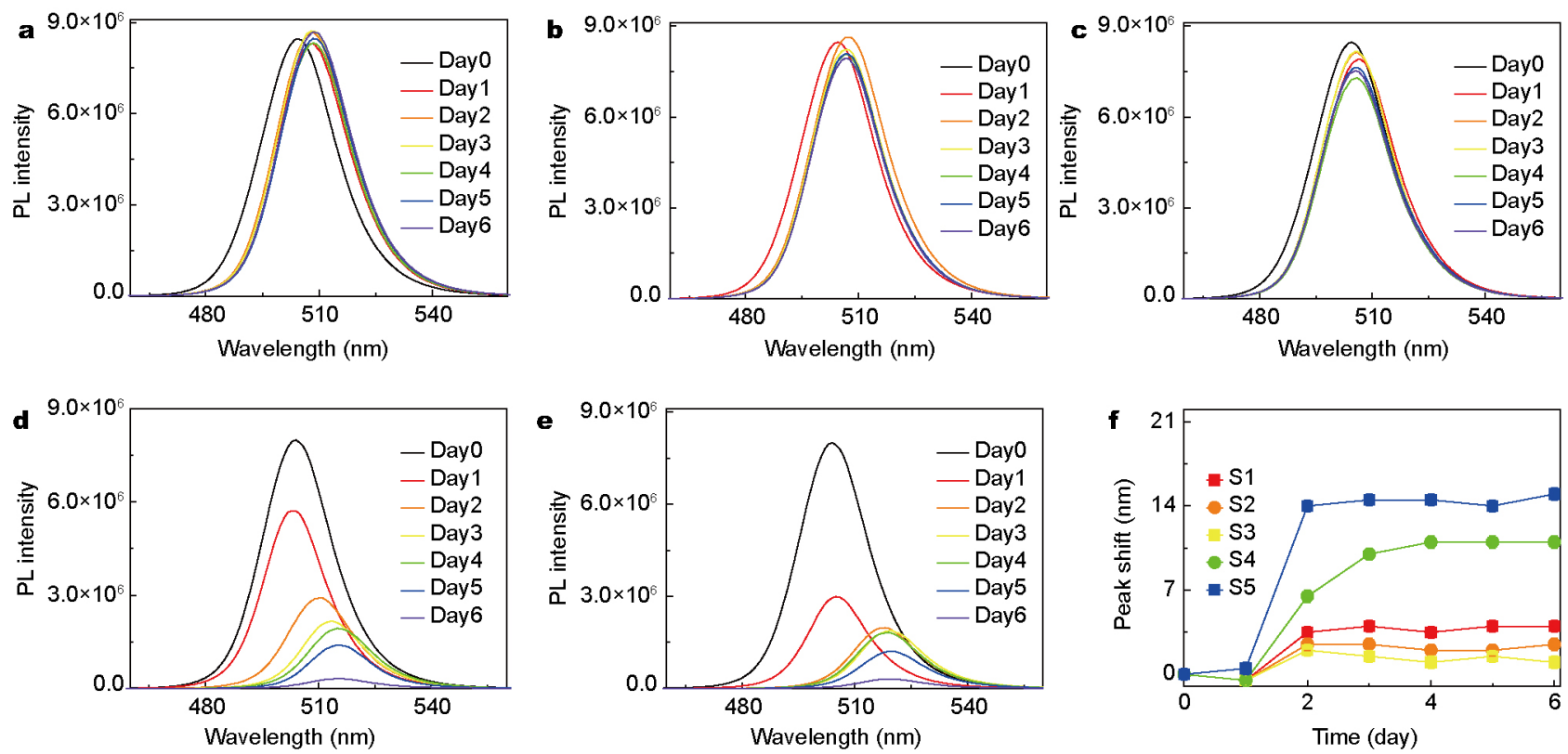

Figure 2 PL emission spectra of samples S1-S5 at different time. (a-e) are corresponding to samples S1-S5, respectively; (f) PL emission peak shift evolution over time for samples S1-S5.

emission of $\mathrm{CsPbBr}_{3}$ QDs. Fig. 2 shows the evolution of PL spectra for samples S1-S5 during the test. Analogous to the absorption spectra, the PL spectra of samples in dark condition keep constant (Figs 2a, b and c corresponding to S1, S2 and S3, respectively). For samples S4 and S5 (Figs $2 \mathrm{~d}$ and e) under light irradiation, the PL intensity decreases drastically. In addition, the emission peaks of these samples also exhibit red shifting over time. Again the red shifts in samples S4 and S5 are more pronounced. Compared with light irradiation, the atmosphere during the storage seems not to influence the long-term stability confirmed by the constant absorption and PL spectra for S1 and S2 in Figs 1 
and 2 .

\section{Photo-degradation $v s$. thermal-degradation}

Light illumination may lead to the degradation of QDs via two main approaches: the first is related to the thermal effect [10], where the vibration excitation by the incident photon or non-radiative recombination of the photo-generated charges can generate heat in the $\mathrm{Cs} \mathrm{PbBr}_{3}$ QDs. The heat dissipation is reported to be efficient in perovskites, which facilitates the thermal-induced mechanical stress and structural change within the QDs [20]. The second process is the photo-chemical reaction in the QDs. It is critical to first distinguish these two different processes in the case of $\mathrm{CsPbBr}_{3}$ QDs.

In order to achieve that, we varied the spectral range of illumination light using color filters in the stability test. We believe that the board white light can induce both thermal effect and photo-chemical reaction, while light with photon energy lower than the band gap can only contribute to thermal process and is unable to generate charges (for details of illumination light to be used see Table 1). We placed a 300-500nm band pass filter for sample S6, which ensures the excitation of QDs by photons with energy higher than the band gap. In the other case, a $550 \mathrm{~nm}$ long pass filter is applied for sample S7, which generates light with photon energy lower than the band gap. As shown in Fig. 3, the samples under board white light illumination (S5) and light with wavelength of 300-500 nm (S6) behave similarly, where red-shifted absorption and emission band together with decreasing absorbance and PL intensity can be observed (Figs $3 \mathrm{a}$ and $\mathrm{d}$ ). On the contrary, when photon energy of the illumination light is lower than the band gap (S7), only the decreasing of PL intensity can be observed (Fig. 3e). This suggests that photo-degradation should be the main origin for the long-term instability of $\mathrm{CsPbrr}_{3}$ QDs. Thermal effect, on the other hand, plays a minor role in the degradation of $\mathrm{CsPbBr}_{3}$ QDs which would not induce any structural damage.

\section{PL dynamics correlated to the photo-stability}

In order to obtain a deeper insight into the photo-degradation process, the PL dynamics of $\mathrm{CsPbBr}_{3}$ QDs during degradation test was studied. We first looked at the change of PL QY as shown in Fig. 4. After 5 days' storage, the PL QYs of samples stored in dark (S1-S3) shows relatively low declining $<20 \%$, while the QYs of samples under light irradiation (S4-S7) decrease up to $40 \%$. In general, the PL QY $(\eta)$ represents the ratio between radiative and non-radiative recombination process in the QDs as:

$$
\eta=\frac{k_{\mathrm{rad}}}{k_{\mathrm{rad}}+k_{\mathrm{nonrad}}}
$$

where $k_{\text {rad }}$ and $k_{\text {nonrad }}$ refer to the rates of the radiative and non-radiative recombination, respectively. Therefore, the
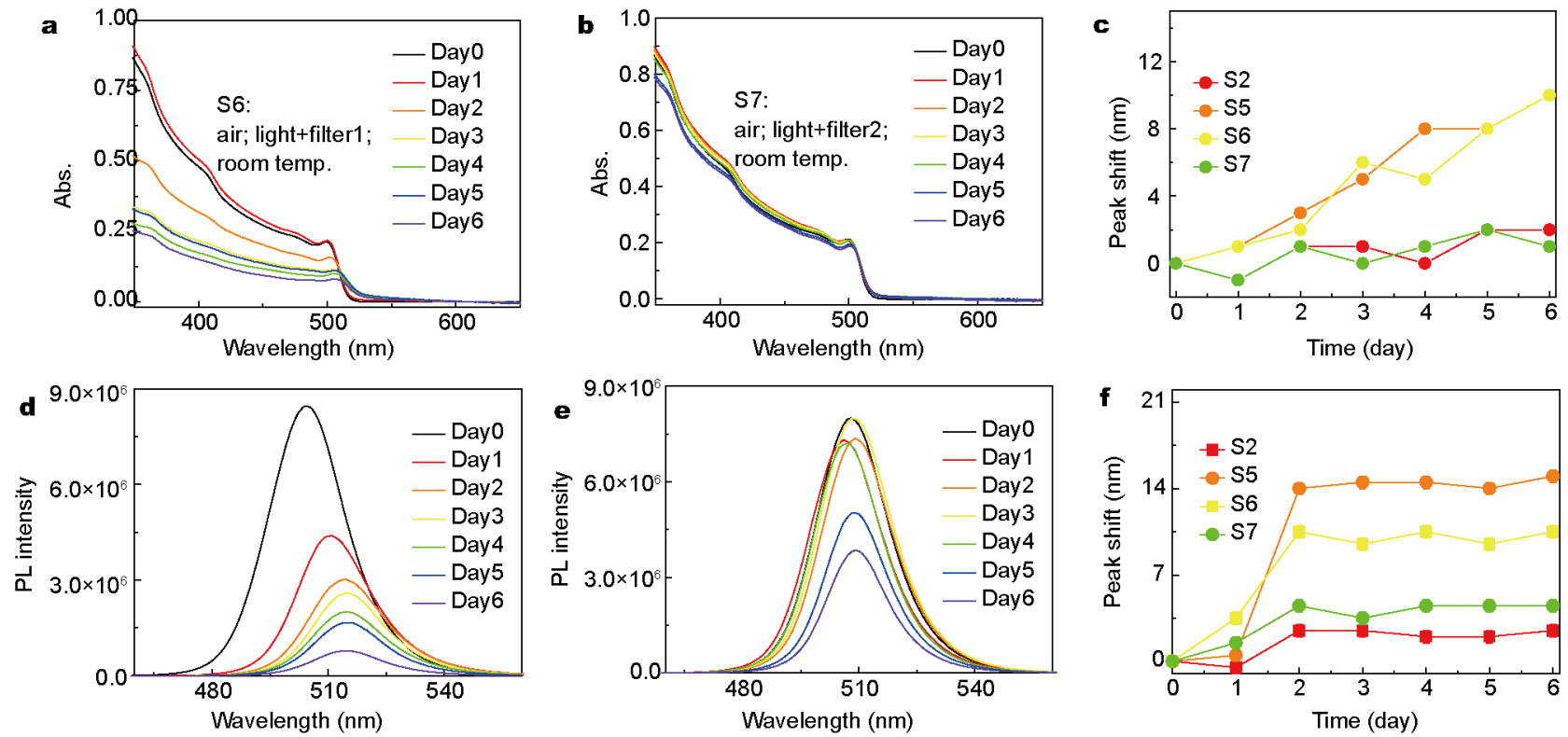

Figure 3 Absorption and PL emission spectra of samples S6 and S7 at different time during the test: (a) absorption spectra of sample S6 (with filter1: 300-500 nm band pass filter), (b) absorption spectra of sample S7 (with filter2: $550 \mathrm{~nm}$ long pass filter), (c) evolution of 1st exciton absorption peaks for sample S2 and S5-S7, (d) PL emission spectra of sample S6, (e) PL emission spectra of sample S7, (f) evolution of PL emission peaks for sample S2 and S5-S7. 


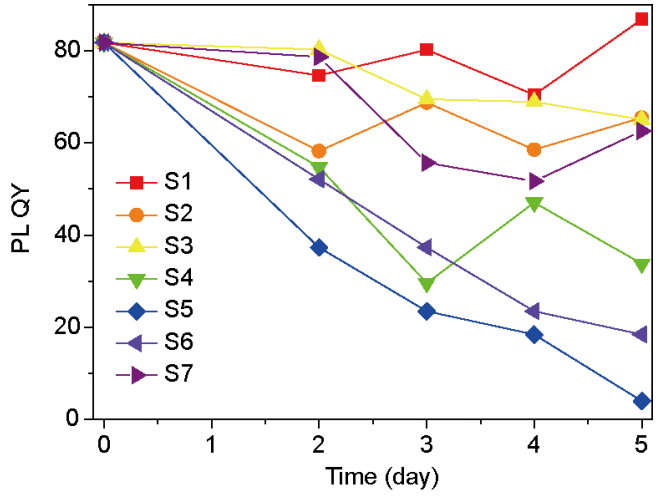

Figure 4 PL QY of samples S1-S7 at different time.

decreasing of PL QY indicates enhanced non-radiative recombination which is usually attributed to the introduction of trap states (especially surface traps) as quenchers in QD materials.

In the next step, we analyzed the emission dynamics by measuring the time-resolved PL decays for samples S2 and S5 (Fig. 5). For sample stored in dark (S2), the PL kinetics remain to be constant single exponential decays with lifetime of $4.2 \pm 0.1 \mathrm{~ns}$ over the entire test process (Fig. 5a) [21]. Such stable PL dynamics is consistent with the steady-state absorption and PL results indicating the absence of intensive degradation. For sample under light irradiation (S5), the PL decays are slowing down as shown in Fig. 5b. We can fit those PL decays by two exponential components as listed in Table 2. The fast component ( $4.2 \mathrm{~ns}$ ) can be attributed to the same intrinsic recombination process as in sample S2. In addition, another slow decay component (43.5 ns) appeared in sample S5 with increasing contribution (i.e., increasing amplitude) during the light illumination as shown in Table 2. According to the above discussion, we can attribute this slow component to the trap emission in the QDs which would be further discussed in the following section.

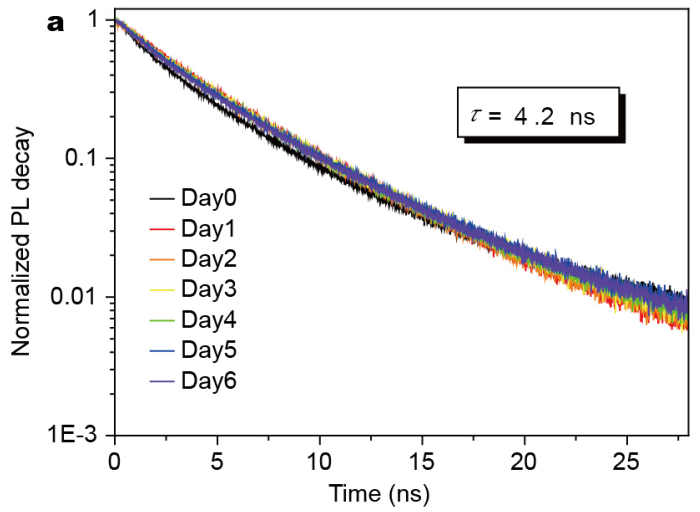

Table 2 Summary of multi-exponential fitting results of PL decays in the sample S5 with the excitation at $438 \mathrm{~nm}$ (at fluence of $0.5 \mu \mathrm{J} \mathrm{cm}^{-2}$ )

\begin{tabular}{ccccc}
\hline $\begin{array}{c}\text { Aging time } \\
\text { (day) }\end{array}$ & $\tau_{1}(\mathrm{~ns})^{\mathrm{a})}$ & $A_{1}(\%)$ & $\tau_{2}(\mathrm{~ns})$ & $A_{2}(\%)$ \\
\hline $0^{\mathrm{b})}$ & 4.2 & 100 & $/$ & 0 \\
1 & 4.2 & $96.2 \pm 0.2$ & $43.5 \pm 0.2$ & $3.8 \pm 0.1$ \\
2 & 4.2 & $87.3 \pm 0.2$. & $43.5 \pm 0.2$ & $12.7 \pm 0.1$ \\
3 & 4.2 & $86.0 \pm 0.2$ & $43.5 \pm 0.2$ & $14.0 \pm 0.1$ \\
4 & 4.2 & $85.1 \pm 0.2$ & $43.5 \pm 0.2$ & $14.9 \pm 0.1$ \\
5 & 4.2 & $84.7 \pm 0.2$ & $43.5 \pm 0.2$ & $15.3 \pm 0.1$ \\
6 & 4.2 & $82.7 \pm 0.2$ & $43.5 \pm 0.2$ & $17.3 \pm 0.1$ \\
\hline
\end{tabular}

a) $\tau_{1}$ was fixed to 4.2 ns during the fitting for test time from 1 to 6 ; b) we used single explanation decay fit for the fresh sample (time $=0$ ).

\section{Models for the photo-degradation process.}

We tried to build up a photo-degradation model of the QDs first based on the structural characterization of the corresponding samples. Fig. 6 shows the light induced structural change studied by XRD and TEM for the sample S2 (kept in dark) and S5 (after light illumination). As shown in TEM images (Figs $6 c$ and d), the $\mathrm{CsPBBr}_{3}$ QDs have initial quasi-cubic morphologies $(9.9 \mathrm{~nm}$ in length, $8.7 \mathrm{~nm}$ in width, see Figs $6 \mathrm{c}$ and d). After light irradiation, the $\mathrm{CsPbBr}_{3}$ QDs tend to aggregate and form elongated nano-rod structures with $61.1 \mathrm{~nm}$ in length and 29.9 $\mathrm{nm}$ in width as shown in Figs 6d and f. In XRD patterns, samples S2 and S5 show different peak width (Figs 6a and b). This also proves the crystal size is increasing in $\mathrm{S} 5$ based on the Scherrer Equation: $D=K \lambda / \beta \cos \theta$, in which $K$ is a constant related to crystallite shape, normally taken as 0.9 , $\lambda$ is the X-ray wavelength ( $\lambda=0.1 \mathrm{~nm}$ in our experiment), $\beta$ is the full width half maximum at corresponding Bragg angle $\theta$. We can then roughly estimate the change of particle size using XRD peaks from (110) plane $\left(2 \theta=21^{\circ}\right)$. The calculated sizes from XRD pattern is close to the TEM

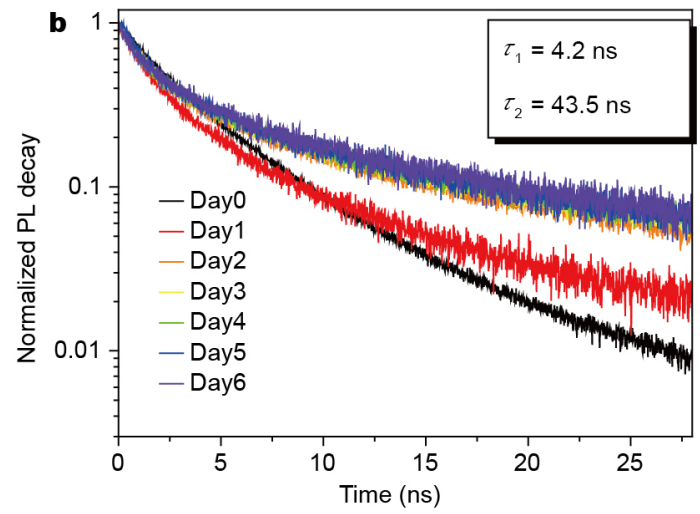

Figure 5 Time-resolved PL decays for sample S2 (a) and S5 (b) at different time, $\lambda_{\text {exc }}=438 \mathrm{~nm}$. PL was detected with a $450 \mathrm{~nm}$ long band pass filter. 

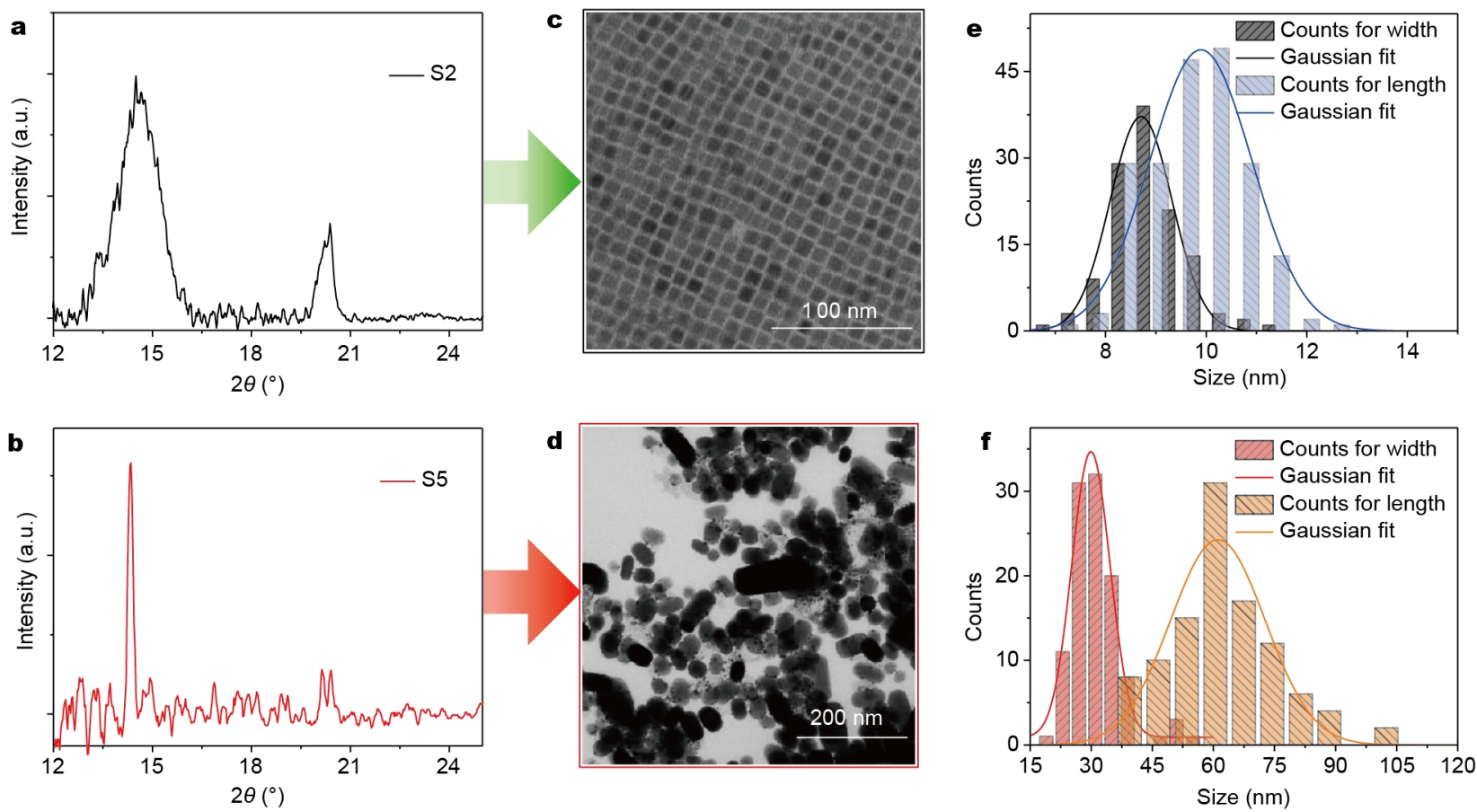

Figure 6 XRD patterns of sample S2 (kept in dark, a) and S5 (after light irradiation, b); TEM images of S2 (c) and S5 (d); the size distribution histograms of $\mathrm{CsPbBr}_{3}$ QDs sample S2 (e) and sample S5 (f) measured along the long edge (length) and the short edge (width).

Table 3 QDs sizes (nm, sample S2 and S5) from TEM images and XRD estimation

\begin{tabular}{ccc}
\hline Samples & TEM & XRD \\
\hline S2 (kept in dark) & $9.9 \pm 1.0 ; 8.7 \pm 1.2$ & $10.4 \pm 0.2$ \\
S5 (after light irradiation) & $61.1 \pm 11.6 ; 29.9 \pm 4.7$ & $37.4 \pm 3.4$ \\
\hline
\end{tabular}

observation as summarized in Table 3. It should be noted that due to the usage of XRD peak from (110) plane in the calculation, we can only evaluate one crystallite dimension in the direction perpendicular to the plane. Based on the results from XRD and TEM, one can conclude the aggregation of QDs and formation of big crystals under light irradiation. This is consistent with the observed red-shifted absorption and PL spectra.

Based on the structural characterization and PL dynamics analysis, we can then propose a mechanism of the photo-induced degradation in $\mathrm{CsPbBr}_{3}$ QDs as below (Fig. 7a). After light excitation, the generated charges have a chance to diffuse to the surface because of the high carrier mobility and fast interfacial charge transfer in $\mathrm{CsPbBr}_{3}$ QDs [22,23]. Once the charges diffuse to the surface, they can be captured by the ionic ligands. As the interaction between $\mathrm{CsPbBr}_{3}$ QDs and the capping ligands is more ionic with highly dynamic ligands binding [24], the ionic ligands are then readily removed from the QDs surface and dissolved into the solvent. As a result, the uncapped surface is formed in QDs leading to the aggregation of neighboring particles. This refers to the red-shifted absorption and PL spectra. When the time of exposure to light is long enough, the precipitation of large QDs clusters would occur corresponding to the observation of scattering in absorption spectra.

The removal of capping agent and aggregation of $\mathrm{CsPBr}_{3}$ QDs would also generate numerous surface/interface dangling bonds which would act as trap sites (Fig. 7a). Fig. 7b demonstrates different excited state dynamics when the trap states are introduced in the QDs. For the as-prepared $\mathrm{CsPbr}_{3}$ QDs after photoexcitation from ground state $(G)$ to excited state $(E)$, they decay to ground state mainly through PL emission with a lifetime of $\sim 4.2$ ns. For the sample after photo-degradation, extra depopulation pathways of excited states occur via trap states. The PL QY data indicate the existence of nonradiative recombination in degraded samples, while the PL decays reveal the occurrence of trap site emission in QDs. In this scenario, we believe there should be both radiative and nonradiative recombination from the trap states in QDs as illustrated in Fig. $7 \mathrm{~b}$.

\section{CONCLUSION}

We studied the stability of $\mathrm{CsPbBr}{ }_{3}$ QDs stored in differ- 

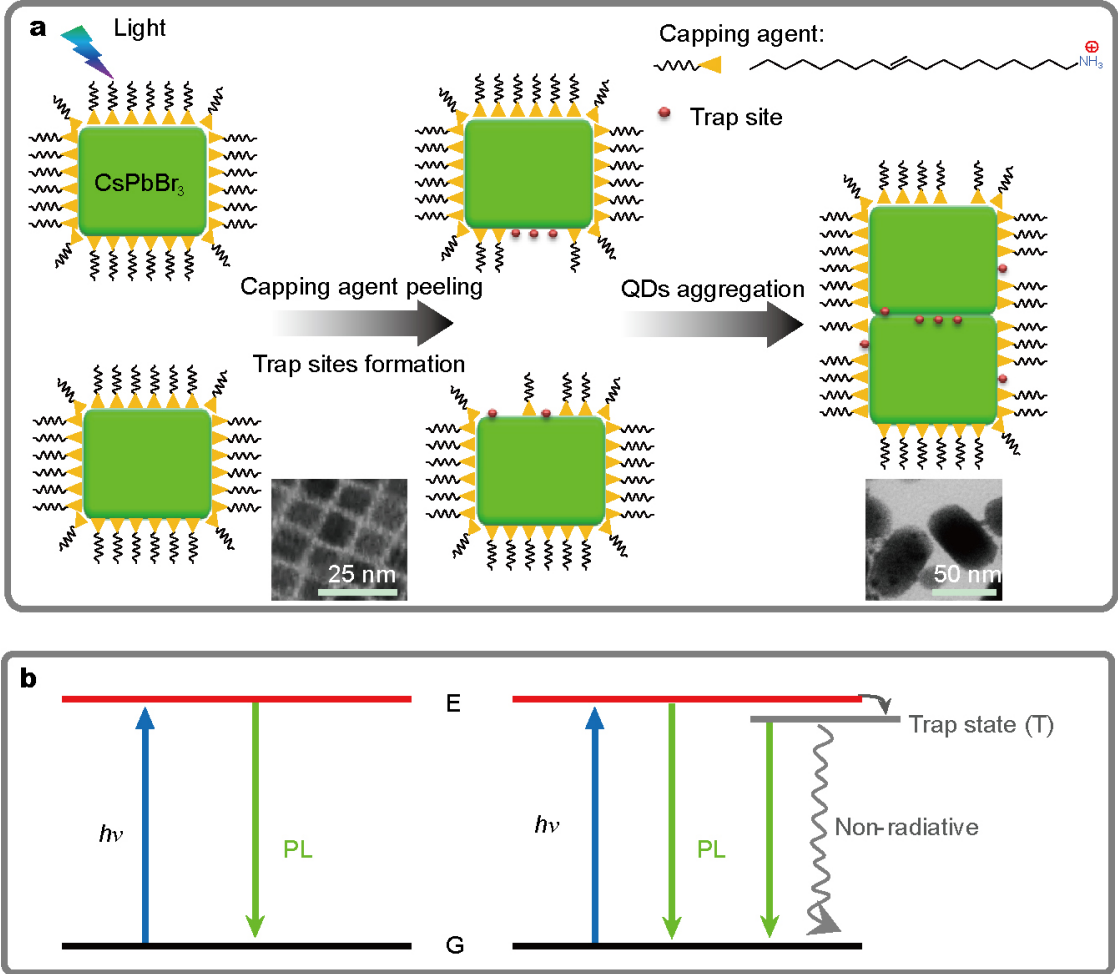

Figure 7 (a) Schematic picture of the photo-degradation pathway occurring on $\mathrm{CsPbBr}_{3}$ QDs in toluene. (b) Mechanism of the various excited-state decay pathways for $\mathrm{CsPbr}_{3}$ QDs.

ent atmosphere, temperature and light conditions. Among those conditions, light illumination plays the most important role in the degradation of the QDs. TEM and XRD characterizations revealed that the long-term light illumination will induce the aggregation and elongation of the nanocrystals, which results in red-shifted absorption and PL spectra of those samples. We also confirmed that such aggregation was induced by the detaching of surface capping agent due to surface charging where the photoexcitation of charges was the critical premise. Based on the PL dynamics studies, it can also be concluded that the photo-induced aggregation of QDs will also introduce both emissive and non-emissive trap states in the QDs. This explains the reduced PL QY together with prolonged PL lifetime after photo-degradation of QDs. Therefore, in order to improve the long term photo-stability of $\mathrm{CsPbBr}_{3}$ QDs for their optical applications, one should try to passivate the surface with tightly bonded agents such as $\mathrm{SiO}_{2}$ or other polymer layers.

Received 26 August 2016; accepted 8 September 2016; published online 20 September 2016

1 Wang Y, Li X, Song J, et al. All-inorganic colloidal perovskite quantum dots: a new class of lasing materials with favorable characteristics. Adv Mater, 2015, 27: 7101-7108
2 Yakunin S, Protesescu L, Krieg F, et al. Low-threshold amplified spontaneous emission and lasing from colloidal nanocrystals of caesium lead halide perovskites. Nat Commun, 2015, 6: 8056

3 Protesescu L, Yakunin S, Bodnarchuk MI, et al. Nanocrystals of cesium lead halide perovskites $\left(\mathrm{CsPb}_{3}, \mathrm{X}=\mathrm{Cl}, \mathrm{Br}\right.$, and I): novel optoelectronic materials showing bright emission with wide color gamut. Nano Lett, 2015, 15: 3692-3696

4 Pan J, Sarmah SP, Murali B, et al. Air-stable surface-passivated perovskite quantum dots for ultra-robust, single- and two-photon-induced amplified spontaneous emission. J Phys Chem Lett, 2015, 6: 5027-5033

5 Nedelcu G, Protesescu L, Yakunin S, et al. Fast anion-exchange in highly luminescent nanocrystals of cesium lead halide perovskites $\left(\mathrm{CsPbX}_{3}, \mathrm{X}=\mathrm{Cl}, \mathrm{Br}, \mathrm{I}\right)$. Nano Lett, 2015, 15: 5635-5640

6 Song J, Li J, Li X, et al. Quantum dot light-emitting diodes based on inorganic perovskite cesium lead halides $\left(\mathrm{CsPbX}_{3}\right)$. Adv Mater, 2015, 27: 7162-7167

7 Li X, Wu Y, Zhang S, et al. $\mathrm{CsPbX}_{3}$ quantum dots for lighting and displays: room-temperature synthesis, photoluminescence superiorities, underlying origins and white light-emitting diodes. Adv Funct Mater, 2016, 26: 2435-2445

8 Ramasamy P, Lim DH, Kim B, et al. All-inorganic cesium lead halide perovskite nanocrystals for photodetector applications. Chem Commun, 2016, 52: 2067-2070

9 Kulbak M, Cahen D, Hodes G. How important is the organic part of lead halide perovskite photovoltaic cells? Efficient $\mathrm{Cs} \mathrm{PbBr}_{3}$ cells. J Phys Chem Lett, 2015, 6: 2452-2456

10 Niu G, Guo X, Wang L. Review of recent progress in chemical stability of perovskite solar cells. J Mater Chem A, 2015, 3: 8970-8980

11 Noel NK, Abate A, Stranks SD, et al. Enhanced photoluminescence 
and solar cell performance via lewis base passivation of organic-inorganic lead halide perovskites. ACS Nano, 2014, 8: 9815-9821

12 Huang S, Li Z, Kong L, et al. Enhancing the stability of $\mathrm{CH}_{3} \mathrm{NH}_{3} \mathrm{PbBr}_{3}$ quantum dots by embedding in silica spheres derived from tetramethyl orthosilicate in "waterless" toluene. J Am Chem Soc, 2016, 138: 5749-5752

13 Akkerman QA, D'Innocenzo V, Accornero S, et al. Tuning the optical properties of cesium lead halide perovskite nanocrystals by anion exchange reactions. J Am Chem Soc, 2015, 137: 10276-10281

14 Chen JS, Zhao GJ, Cook TR, et al. Photophysical properties of selfassembled multinuclear platinum metallacycles with different conformational geometries. J Am Chem Soc, 2013, 135: 6694-6702

15 Brannon JH, Magde D. Absolute quantum yield determination by thermal blooming. Fluorescein. J Phys Chem, 1978, 82: 705-709

16 Chen J, Žídek K, Abdellah M, et al. Surface plasmon inhibited photo-luminescence activation in CdSe/ZnS core-shell quantum dots. J Phys-Condens Matter, 2016, 28: 254001

17 Stoumpos CC, Malliakas CD, Peters JA, et al. Crystal growth of the perovskite semiconductor $\mathrm{Cs} \mathrm{PbBr}_{3}$ : a new material for high-energy radiation detection. Cryst Growth Des, 2013, 13: 2722-2727

18 Koolyk M, Amgar D, Aharon S, et al. Kinetics of cesium lead halide perovskite nanoparticle growth; focusing and de-focusing of size distribution. Nanoscale, 2016, 8: 6403-6409

19 Cottingham P, Brutchey RL. On the crystal structure of colloidally prepared $\mathrm{CsPbBr}_{3}$ quantum dots. Chem Commun, 2016, 52: 5246-5249

20 Pisoni A, Jaćimović J, Barišić OS, et al. Ultra-low thermal conductivity in organic-inorganic hybrid perovskite $\mathrm{CH}_{3} \mathrm{NH}_{3} \mathrm{PbI}_{3}$. J Phys Chem Lett, 2014, 5: 2488-2492

21 Makarov NS, Guo S, Isaienko O, et al. Spectral and dynamical properties of single excitons, biexcitons, and trions in cesium-lead- halide perovskite quantum dots. Nano Lett, 2016, 16: 2349-2362

22 Yettapu GR, Talukdar D, Sarkar S, et al. Terahertz conductivity within colloidal $\mathrm{Cs} \mathrm{PbBr}_{3}$ perovskite nanocrystals: remarkably high carrier mobilities and large diffusion lengths. Nano Lett, 2016, 16 : 4838-4848

23 Wu K, Liang G, Shang Q, et al. Ultrafast interfacial electron and hole transfer from $\mathrm{CsPbBr}_{3}$ perovskite quantum dots. J Am Chem Soc, 2015, 137: 12792-12795

24 De Roo J, Ibáñez M, Geiregat P, et al. Highly dynamic ligand binding and light absorption coefficient of cesium lead bromide perovskite nanocrystals. ACS Nano, 2016, 10: 2071-2081

Acknowledgments This work was supported by the National Priorities Research Program from the Qatar National Research Fund (a member of Qatar Foundation) (NPRP7-227-1-034), the Swedish Foundation for International Cooperation in Research and Higher Education (STINT, CH2015-6232), the Academy of Finland, and the Program of Study Abroad for Young Teachers by Agricultural University of Hebei. We thank Jasper Rikkert Plaisier for his support in XRD measurement. Collaboration within NanoLund is acknowledged.

Author contributions Chen J conceived and conducted the experiments, analyzed the results and wrote the paper; Zheng $\mathrm{K}$ supervised the project, conceived the experiments, analyzed the results and revised the paper. Liu D prepared the samples, conducted the experiments and collected the data. Nuuttila $\mathrm{L}$ and Lehtivuori $\mathrm{H}$ conducted the sample TEM charterization. Al-Marri MJ involved in the experimental design and paper writing. All authors contributed to the general discussion.

Conflict of interest The authors declare that they have no conflict of interest. 

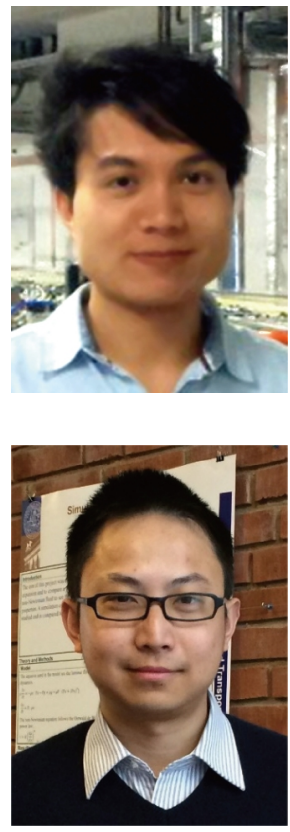

Junsheng Chen received his BSc degrees in materials physics and management from China University of Petroleum (Huadong) in 2010. Now he is a joint PhD candidate of Lund University and Dalian Institute of Chemical Physics, Chinese Academy of Sciences. His research interests include semiconductor nanocrystals preparation and excited state dynamic processes in semiconductor nanocrystals and organic supramolecules.
Kaibo Zheng received his $\mathrm{PhD}$ degree from the Department of Material Science, Fudan University in 2010. He is now a senior researcher at the Division of Chemical Physics, Lund University, Sweden. His research interests include design, structural characterization and ultrafast photo-induced dynamics in nanostructured optoelectronic materials such as semiconductor quantum dots, nanowires and perovskite materials.

\section{光电器件用 $\mathrm{CsPbBr}_{3}$ 钻钛矿量子点的光稳定性研究}

陈俊生 ${ }^{1,2}$, 刘东州 ${ }^{3,4}$, MohammedJ. Al-Marri ${ }^{5}$, LauriNuuttila $^{6}$, HeliLehtivuori $^{6}$, 郑凯波 $1,5^{*}$

摘要 全无机 $\mathrm{CsPBr}_{3}$ 钙钛矿量子点因其优异的发光性能, 而在太阳能电池、发光二极管、光探头和激光器件等应用研究领域受到广泛关 注. 基于此, 我们研究了 $\mathrm{CsPbr}_{3}$ 钙钛矿量子点在不同光照、气氛和温度条件下的稳定性. 光照下, 量子点表面缺陷态的产生和量子点的聚 集促使量子点的发光效率降低, 吸收和发光光谱发生红移. 透射电子显微镜和X-光单晶衍射实验结果表明, 在光照下量子点发生了聚集 进而形成了大尺寸的晶体. 我们的工作表明光照是影响 $\mathrm{CsPBr}_{3}$ 钲钛矿量子点稳定性的决定性因素, 因此需要改善其光稳定性从而优化 $\mathrm{CsPBr}_{3}$ 钻钛矿量子点光电器件性能. 